\title{
REMARKS ON $\mathbb{A}^{1}$-HOMOTOPY GROUPS OF SMOOTH TORIC MODELS
}

\author{
ARAVIND ASOK
}

\begin{abstract}
We extend previous results on $\mathbb{A}^{1}$-homotopy groups of smooth proper toric varieties to the case of smooth proper toric models, i.e., smooth proper equivariant compactifications of possibly non-split tori, in characteristic 0 .
\end{abstract}

\section{Statement of results}

Fix a field $k$ having characteristic 0 , and let $\mathcal{S} m_{k}$ denote the category of schemes that are separated, smooth and have finite type over $k$. Suppose $X$ is a smooth proper $k$-scheme. Let $\mathcal{H}(k)$ denote the $\mathbb{A}^{1}$-homotopy category of $k$-schemes as constructed in [8, §3.2]. Assume $X(k)$ is non-empty, and fix $x \in X(k)$. One can study the $\mathbb{A}^{1}$-homotopy sheaves of groups $\pi_{i}^{\mathbb{A}^{1}}(X, x)$ (the Nisnevich sheaves of groups on $\mathcal{S} m_{k}$ denoted $a \underline{\pi}_{i}^{\mathbb{A}^{1}}(X, x)$ on $[8$, p. 110$\left.]\right)$. Our aim in this short note is to show that the "geometric" decomposition of $\mathbb{A}^{1}$-homotopy sheaves of groups of smooth proper "split" toric varieties, i.e., equivariant compactifications of $\mathbb{G}_{m}{ }^{\times n}$, studied in [1] and [9] extends to "non-split" toric varieties, i.e., equivariant compactifications of tori $T$ over $k$. We will refer to equivariant compactifications of tori $T$ over $k$ as toric $T$-models [6, §5].

Let $\bar{k}$ denote a fixed algebraic closure of $k$ and let $G_{k}$ denote the Galois group of $\bar{k}$ over $k$. For a $k$-scheme $Y$, let $Y_{\bar{k}}$ denote the variety obtained by extending scalars to $\bar{k}$. Suppose $X$ is a smooth proper toric $T$-model. One knows that $\operatorname{Pic}\left(X_{\bar{k}}\right)$ is a finitely generated $G_{k}$-module, and we denote the associated dual $k$-torus - the Neron-Severi torus - by $T_{N S(X)}$. With any toric $T$-model, one can associate a fan $\Sigma$ in $X^{*}\left(T_{\bar{k}}\right)$ that is $G_{k}$-invariant. Cox's construction [5] realizing any "split" smooth proper toric variety as a geometric quotient of an open subscheme of affine space by a free action of $T_{N S(X)}$ can be generalized to the non-split case: if $X$ is a smooth proper toric $T$-model, there are a $T_{N S(X)}$-torsor $f: U \rightarrow X$ and an open immersion $U \hookrightarrow \mathbb{A}_{k}^{n}\left(n=\operatorname{dim} T+\operatorname{dim} T_{N S}(X)\right)$ [6, Proposition 5.6]. Let $\mathcal{H}_{\text {ét }}^{1}\left(T_{N S(X)}\right)$ denote the Nisnevich sheafification of the presheaf (on $\left.\mathcal{S} m_{k}\right) U \mapsto H_{\text {ét }}^{1}\left(U, T_{N S(X)}\right)$.

Theorem 1.1. Assume $k$ is a field having characteristic 0 and $T$ is a $k$-torus. Suppose $X$ is a smooth proper toric $T$-model, and let $x$ denote the $k$-rational point of $X$ corresponding to $1 \in T(k)$. The $T_{N S(X)}$-torsor $f: U \rightarrow X$ above is an $\mathbb{A}^{1}$-cover. In particular, if $\tilde{x}$ is any lift of $x$, there is a short exact sequence (of Nisnevich sheaves of groups)

$$
1 \longrightarrow \pi_{1}^{\mathbb{A}^{1}}(U, \tilde{x}) \longrightarrow \pi_{1}^{\mathbb{A}^{1}}(X, x) \longrightarrow T_{N S(X)} \longrightarrow 1,
$$

Received by the editors October 28, 2009.

Aravind Asok was partially supported by National Science Foundation Award DMS-0900813. 
and, for each integer $i>1$, there are isomorphisms $\pi_{i}^{\mathbb{A}^{1}}(U, \tilde{x}) \stackrel{\sim}{\rightarrow} \pi_{i}^{\mathbb{A}^{1}}(X, x)$. Finally, $f$ induces a morphism of sheaves $\pi_{0}^{\mathbb{A}^{1}}(X) \rightarrow \mathcal{H}_{e ́ t}^{1}\left(T_{N S(X)}\right)$ that is an isomorphism on sections over finitely generated separable extensions $L / k$.

Remark 1.2. There are examples of $k$-tori $T$ and smooth proper toric $T$-models $X$ for which $\pi_{0}^{\mathbb{A}^{1}}(X)(k)$ is non-trivial. Thus, over non separably closed fields, we have the interesting phenomenon that a smooth proper $\mathbb{A}^{1}$-disconnected space can have $\mathbb{A}^{1}$ connected covering spaces! For a manifestation of this phenomenon for non-proper smooth varieties, one can consider the morphism $\mathbb{A}^{m} \backslash 0 \rightarrow \mathbb{A}^{m} \backslash 0 / \mu_{n}$ [1, Remark $3.13]$.

\section{Torus torsors as $\mathbb{A}^{1}$-covering spaces}

The word space, will mean "object of $\Delta^{\circ} \mathcal{S} h v_{N i s}\left(\mathcal{S} m_{k}\right)$ " (the category of simplicial Nisnevich sheaves on $\left.\mathcal{S} m_{k}\right)$; we use caligraphic letters (e.g., $\left.\mathcal{X}, \mathcal{Y}\right)$ to denote such objects. We set $[\mathcal{X}, \mathcal{Y}]_{s}:=\operatorname{hom}_{\mathcal{H}_{s}\left(\left(\mathcal{S} m_{k}\right)_{N i s}\right)}(\mathcal{X}, \mathcal{Y})$, where $\mathcal{H}_{s}\left(\left(\mathcal{S} m_{k}\right)_{N i s}\right)$ is as on 8 , p. 49] and $[\mathcal{X}, \mathcal{Y}]_{\mathbb{A}^{1}}:=\operatorname{hom}_{\mathcal{H}(k)}(\mathcal{X}, \mathcal{Y})$. A morphism $f: \mathcal{X} \rightarrow \mathcal{Y}$ of $k$-spaces is an $\mathbb{A}^{1}$-cover ( $c f$. [7, Section 4.1]) if it has the unique right lifting property with respect to morphisms that are simultaneously $\mathbb{A}^{1}$-weak equivalences and monomorphisms of sheaves, i.e., $\mathbb{A}^{1}$-acyclic cofibrations.

Proposition 2.1. Let $T$ be a multiplicative group over a field $k$ having characteristic 0 . If $X$ is a smooth scheme, and $\pi: U \rightarrow X$ is a T-torsor locally trivial in the étale topology, then $\pi$ is an $\mathbb{A}^{1}$-cover and, in particular, an $\mathbb{A}^{1}$-fibration.

Let $B T$ denote the simplicial classifying space of $T$ viewed as a Nisnevich sheaf of groups, and let $B T_{\text {ét }}$ denote the simplicial classifying space of $T$ viewed as an étale sheaf of groups. Let $\alpha:\left(\mathcal{S} m_{k}\right)_{\text {ét }} \rightarrow\left(\mathcal{S} m_{k}\right)_{N i s}$ be the morphism of sites induced by the identity functor. Set $B_{\text {ét }} T:=\mathbf{R} \alpha_{*} B T_{\text {ét }}$; see [8, $\left.\S 4.1\right]$ for more details.

Lemma 2.2 ( $c f$. [2, Lemma 4.2.4]). The space $B_{\text {ét }} T$ is $\mathbb{A}^{1}$-local.

Proof. By adjunction, one has canonical bijections

$$
\operatorname{hom}_{\mathcal{H}_{s}\left(\left(\mathcal{S} m_{k}\right)_{N i s}\right)}\left(U, B_{\text {ét }} T\right) \stackrel{\sim}{\longrightarrow} \operatorname{hom}_{\mathcal{H}_{s}^{e ́ t}(k)}\left(U, B T_{\text {ét }}\right) .
$$

Choosing a fibrant model for $B T_{\text {ét }}$, and using [8, §2 Proposition 3.19 and $\S 4$ Proposition 1.16], to check that $B_{\text {ét }} T$ is $\mathbb{A}^{1}$-local, it suffices to prove that that the maps

$$
H_{\text {ét }}^{i}(U, T) \longrightarrow H_{\text {ét }}^{i}\left(U \times \mathbb{A}^{1}, T\right)
$$

are bijections for $i=0,1$. For $i=0$, this a consequence of étale descent: if $k^{\prime} / k$ is a separable extension splitting $T$, then it suffices to observe that any morphism $U \times \mathbb{A}^{1} \rightarrow \mathbb{G}_{m}{ }^{\times n}$ factors through a morphism $U \rightarrow \mathbb{G}_{m}{ }^{\times n}$. For $i=1$ one could apply 2, Lemma 4.3.7 and Proposition 4.4.3]. For a direct proof, observe that 4 Lemma 2.4], establishes the result for affine $X$ (Grothendieck showed that étale and flat cohomology coincide Ibid. p.159). We reduce the case of general $X$ to the affine case by comparing the exact sequences of low degree terms for the Leray spectral sequences associated with an open affine cover $u: U \rightarrow X$ and the corresponding cover $u \times i d: U \times \mathbb{A}^{1} \rightarrow X \times \mathbb{A}^{1}$. 
Proof of Proposition 2.1. After Lemma 2.2 the proof is essentially [7, Lemma 4.5(2)]; here are the details. Start with an $\mathbb{A}^{1}$-acyclic cofibration $j: \mathcal{A} \rightarrow \mathcal{B}$ fitting into a diagram

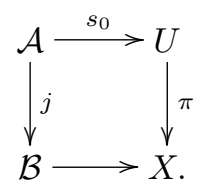

Now, since $B_{\text {ét }} T$ is $\mathbb{A}^{1}$-local, the natural maps $\left[\mathcal{B}, B_{\text {ét }} T\right]_{s} \rightarrow\left[\mathcal{A}, B_{\text {ét }} T\right]_{s}$ and $[\mathcal{B}, T]_{s} \rightarrow$ $[\mathcal{A}, T]_{s}$ are bijections. The pullback of $\pi$ to $\mathcal{A}$ admits a section and is therefore a trivial torsor. By the first bijection just mentioned, it follows that the pullback of $\pi$ to $\mathcal{B}$ is also trivial, and thus also admits a section, which we denote by $s$. The composite morphism $j \circ s$ need not be equal to $s_{0}$, but if it is not, then there is an element $t_{0} \in[\mathcal{A}, T]_{s}$ such that $t_{0} \cdot s=s_{0}$. By the second bijection mentioned at the beginning of this paragraph, the element $t_{0}$ determines a unique element $t$ of $[\mathcal{B}, T]_{s}$. The product $t^{-1} \cdot s$ is a new section of $\pi$ pulled back to $\mathcal{B}$. By construction this new section gives back $s_{0}$ upon restriction to $\mathcal{A}$ and thus provides the necessary (unique) lift.

Proof of Theorem 1.1. We return to the notation of the introduction: $X$ is a smooth proper toric $T$-model, $T_{N S(X)}$ is the associated Neron-Severi torus and $f: U \rightarrow X$ is the $T_{N S(X)}$-torsor constructed in [6, Proposition 5.6].

Since $X$ is proper, it follows from, e.g., [5, Lemma 1.4] that $U$ has complement of codimension $\geq 2$ in the affine space in which it sits since the same thing is true upon passing to a separable closure. Since $k$ has characteristic 0 and is thus infinite, it follows that $U$ is even connected by lines. (In fact, [1, Proposition 5.12] gives conditions guaranteeing that this complement has codimension $\geq d$, depending only on the fan of $X_{\bar{k}}$.) In any case, we can choose a point $\tilde{x}$ lifting $x$.

By Proposition 2.1 $\pi$ is an $\mathbb{A}^{1}$-cover and thus an $\mathbb{A}^{1}$-fibration. Consider the long exact sequence in $\mathbb{A}^{1}$-homotopy groups of $\pi$, which exists by a formal argument in the theory of model categories (cf. [1, Remark 3.2]). The higher $(i>1)$ homotopy (sheaves of) groups of $B_{\text {ét }} T_{N S(X)}$ are trivial, and $\pi_{1}^{\mathbb{A}^{1}}\left(B_{\text {ét }} T_{N S(X)}\right)=T_{N S(X)}$ (again, see [8, $\S 4$ Proposition 1.16]). We then have a long exact sequence of groups (and pointed sets)

$$
\begin{aligned}
1 \longrightarrow \pi_{1}^{\mathbb{A}^{1}}(U, \tilde{x}) \longrightarrow \pi_{1}^{\mathbb{A}^{1}}(X, x) \longrightarrow T_{N S(X)} \\
\longrightarrow \pi_{0}^{\mathbb{A}^{1}}(U) \longrightarrow \pi_{0}^{\mathbb{A}^{1}}(X) \longrightarrow \pi_{0}^{\mathbb{A}^{1}}\left(B_{\text {ét }} T_{N S(X)}\right),
\end{aligned}
$$

and for each $i>1$, we have isomorphisms $\pi_{i}^{\mathbb{A}^{1}}(U, \tilde{x}) \stackrel{\sim}{\rightarrow} \pi_{i}^{\mathbb{A}^{1}}(X, x)$.

For the case $i=0$, observe that the morphism $X \rightarrow B_{\text {ét }} T_{N S(X)}$ classifying $f$ induces the morphism $\pi_{0}^{\mathbb{A}^{1}}(X) \rightarrow \pi_{0}^{\mathbb{A}^{1}}\left(B_{\text {ét }} T_{N S(X)}\right)$. Using the $\mathbb{A}^{1}$-weak equivalence $X \rightarrow \operatorname{Sing}_{*}^{\mathbb{A}^{1}}(X)$, there is an induced epimorphism $\pi_{0}^{s}\left(\operatorname{Sing}_{*}^{\mathbb{A}^{1}}(X)\right) \rightarrow \pi_{0}^{\mathbb{A}^{1}}(X)$ by $[\underline{8}, \S 2$ Corollary 3.22]. Again using the fact that $X$ is proper, we conclude $\pi_{0}^{s}\left(\operatorname{Sing}_{*}^{\mathbb{A}^{1}}(X)\right)(L)$ is $X(L) / R$.

Since $B_{\text {ét }} T_{N S(X)}$ is $\mathbb{A}^{1}$-local, $\pi_{0}^{\mathbb{A}^{1}}\left(B_{\text {ét }} T\right)=\mathcal{H}_{\text {ét }}^{1}\left(T_{N S(X)}\right)$. Taking sections over finitely generated separable extensions $L / k$ determines a morphism of functors (on 
field extensions)

$$
X(L) / R \longrightarrow H_{\text {ét }}^{1}\left(L, T_{N S}(X)\right)
$$

that coincides with the "obvious" such morphism gotten by restricting the $T_{N S}(X)$ torsor $f: U \rightarrow X$ to $L$-points of $X$. The torus $T_{N S(X)}$ is flasque (see, e.g., 3, Proposition 6]) so [3, §5 Corollaire 1] implies that the restriction map $X(L) / R \rightarrow$ $H_{\text {ét }}^{1}\left(L, T_{N S}(X)\right)$ is a bijection. It follows that $\pi_{0}^{\mathbb{A}^{1}}(X) \rightarrow \mathcal{H}_{\text {ét }}^{1}\left(T_{N S(X)}\right)$ is an isomorphism on sections over separable finitely generated $L / k$.

Remark 2.3. The statement in Theorem 1.1 involving $\pi_{0}^{\mathbb{A}^{1}}$ provides an alternate proof of [2, Theorem 2.4.3] in the special case of smooth proper toric models. Furthermore, this statement can be strengthened slightly. Indeed, the multiplication morphism $T \times T \rightarrow T$ gives rise to a rational map $X \times X \rightarrow X$. Resolving indeterminacy, we get a morphism $X^{\prime} \rightarrow X \times X$ (that is a composite of blow-ups). One can check that this induces a composition on $\pi_{0}^{\mathbb{A}^{1}}(X)(L)$ for any $L / k$ (coinciding with the composition on $R$-equivalence classes). The map of the proposition is in fact a homomorphism of abelian groups. One would like to show that $\pi_{0}^{\mathbb{A}^{1}}(X)$ can be equipped with the structure of a Nisnevich sheaf of abelian groups and that the map $\pi_{0}^{\mathbb{A}^{1}}(X) \rightarrow \mathcal{H}_{\text {ét }}^{1}\left(T_{N S(X)}\right.$ is an isomorphism of sheaves.

\section{References}

[1] A. Asok and B. Doran, $\mathbb{A}^{1}$-homotopy groups, excision and solvable quotients, Adv. Math. 221 (2009), no. 4, 1144-1190. $353,354,355$

[2] A. Asok and F. Morel, Smooth varieties up to $\mathbb{A}^{1}$-homotopy and algebraic h-cobordisms (2010) . Preprint available at http://arxiv.org/abs/0810.0324 354356

[3] J.-L. Colliot-Thélène and J.-J. Sansuc, La R-équivalence sur les tores, Ann. Sci. École Norm. Sup. (4) 10 (1977), no. 2, 175-229. 356

[4] - Principal homogeneous spaces under flasque tori: applications, J. Algebra 106 (1987), no. $1,148-205.354$

[5] D. A. Cox, The homogeneous coordinate ring of a toric variety, J. Algebraic Geom. 4 (1995), no. $1,17-50.353,355$

[6] A. S. Merkurjev and I. A. Panin, $K$-theory of algebraic tori and toric varieties, $K$-Theory 12 (1997), no. 2, 101-143. 353355

[7] F. Morel, $\mathbb{A}^{1}$-algebraic topology over a field (2006) Preprint, available at http://www . mathematik. uni-muenchen.de/ morel/preprint.html 354355

[8] F. Morel and V. Voevodsky, $\mathbb{A}^{1}$-homotopy theory of schemes, Inst. Hautes Études Sci. Publ. Math. (1999), no. 90, 45-143 (2001). 353 354355

[9] M. Wendt, On the $\mathbb{A}^{1}$-fundamental groups of smooth toric varieties, Adv. Math. 223 (2010), no. $1,352-378.353$

Department of Mathematics, University of Southern California, Los Angeles, CA 90089-2532

E-mail address: asok@usc.edu 